\title{
EXTRACTION OF TRIPLE GAUGE COUPLINGS (TGC'S)
}

Revised February 2002 by C. Caso (University of Genova) and A. Gurtu (Tata Institute).

Fourteen independent couplings, 7 each for $Z W W$ and $\gamma W W$, completely describe the $V W W$ vertices within the most general framework of the electroweak Standard Model (SM) consistent with Lorentz invariance and $\mathrm{U}(1)$ gauge invariance. Of each of the 7 TGC's, 3 conserve $C$ and $P$ individually, 3 violate $C P$, and one TGC violates $C$ and $P$ individually while conserving $C P$. Assumption of $C$ and $P$ conservation and electromagnetic gauge invariance reduces the independent $V W W$ couplings to five: one common set [1,2] is $\left(\Delta \kappa_{\gamma}, \Delta \kappa_{Z}, \lambda_{\gamma}, \lambda_{Z}, \Delta g_{1}^{Z}\right)$, where $\Delta \kappa_{\gamma}=\Delta \kappa_{Z}=\Delta g_{1}^{Z}=0$ and $\lambda_{\gamma}=\lambda_{Z}=0$ in the Standard Model at the tree level. The $W$ magnetic dipole moment, $\mu_{W}$, and the $W$ electric quadrupole moment, $q_{W}$, are expressed as $\mu_{W}=e\left(1+\kappa_{\gamma}+\lambda_{\gamma}\right) / 2 M_{W}$ and $q_{W}=-e\left(\kappa_{\gamma}-\lambda_{\gamma}\right) / M_{W}^{2}$.

Precision measurements of suitable observables at LEP1 has already led to an exploration of much of the TGC parameter space. For LEP2 data, the LEP Collaborations have agreed to express their results in terms of the parameters $\Delta g_{1}^{Z}, \Delta \kappa_{\gamma}$ and $\lambda_{\gamma}\left(\lambda_{Z}\right.$ and $\Delta \kappa_{Z}$ are related to these by gauge invariance).

At LEP2 the $V W W$ coupling arises in $W$-pair production via $s$-channel exchange or in single $W$ production via the radiation of a virtual photon off the incident $e^{+}$or $e^{-}$. At the TEVATRON hard photon bremstrahlung off a produced $W$ or $Z$ signals the presence of a triple gauge vertex. In order to extract the value of one TGC the others are generally kept fixed to their SM values.

\section{References}

1. K. Hagiwara et al., Nucl. Phys. B282, 253 (1987).

2. G. Gounaris et al., CERN 96-01 525. 